\title{
EVALUACIÓN DE UNA TÉCNICA DE PROTECCIÓN FÍSICA EN EL ÉXITO DE ESTABLECIMIENTO DE DOS ESPECIES LEÑOSAS NATIVAS EN ZONAS CON HORMIGAS CORTADORAS
}

\author{
EVALUATION OF A PHYSICAL PROTECTION TECHNIQUE IN THE ESTABLISHMENT \\ SUCCESS OF TWO WOODY NATIVE SPECIES IN AREAS WITH LEAF CUTTER ANTS
}

\author{
Julian Arriaga ${ }^{1 *}\left(\mathbb{D}\right.$, Romina C. Torres ${ }^{1,2,3}$ y Daniel Renison ${ }^{1,2,3}$
}

1. Centro de Ecología y Recursos Naturales Renovables Dr. Ricardo Luti. Facultad de Ciencias Exactas, Físicas y Naturales. Universidad Nacional de Córdoba. Av. Vélez Sarsfield 1611, X5016GCA Córdoba, Argentina.

2. Instituto de Investigaciones Biológicas y Tecnológicas (CONICET - Universidad Nacional de Córdoba). Av. Vélez Sarsfield 1611, X5016GCA Córdoba, Argentina.

3. ONG Ecosistemas Argentinos. 27 de abril 2050, Córdoba, Argentina.

*arriagaj823@gmail.com

Citar este artículo ARRIAGA, J., R. C. TORRES \& D. RENISON. 2021. Evaluación de una técnica de protección física en el éxito de establecimiento de dos especies leñosas nativas de las Sierras de Córdoba (Argentina) en zonas con hormigas cortadoras. Bol. Soc. Argent. Bot. 56: 187-199.

DOI: https://doi. org/10.31055/1851.2372.v56. n2.32100

Recibido: 9 Feb 2021

Aceptado: 13 May 2021

Publicado en línea: 10 Jun 2021

Publicado impreso: 30 Jun 2021

Editor: Ramiro Aguilar

ISSN versión impresa 0373-580X ISSN versión on-line 1851-2372

\section{SUMmARY}

Background and aims: The restoration of forests in areas degraded by anthropic activities is an important practice in the management of mountain systems. Much research is yet needed on methods to reduce effects of herbivores and harsh environments on the establishment of planted tree saplings. Here, we evaluate the effectiveness of a physical protection using polyvinyl chloride (PVC) cylinders in the plantation of two native tree species in the mountains of Central Argentina, in an area with the presence of leaf-cutting ants.

M\&M: The study species were escallonia (Escallonia cordobensis) and maitén (Maytenus boaria). The plantation site was at $1840 \mathrm{~m}$ asl. We used 230 seedlings per species, 100 with and 130 without a PVC protection. Outplanting was performed during the wet season in areas with low vegetation. We measured height, survival and herbivory every six months for two years.

Results: For escallonia and maitén, respectively, physical protections increased biannual survival in 24 and $26 \%$, increased height in 7.3 and $12.6 \mathrm{~cm}$, and reduced herbivory by leaf-cutting ants in 30 and $51 \%$ as compared to non-protected saplings.

Conclusions: The favorable effects of PVC protections on survival and growth could be due to the decrease in herbivory caused by leaf-cutting ants and also due to the protection against harsh abiotic conditions. We advocate for longer term studies which also aim at understanding the mechanisms underlying the improvement in seedling establishment.

\section{KEY WORDS}

Acromyrmex lobicornis, Argentina, Córdoba, Escallonia cordobensis, Maytenus boaria, reforestation.

\section{RESUMEN}

Introducción y objetivos: La restauración de bosques en sitios degradados por actividades antrópicas es una actividad importante en el manejo de sistemas montanos. Aún hace falta investigación tendiente a reducir el efecto de la herbivoría y de las condiciones ambientales hostiles sobre el establecimiento de plantines de árboles. Nos propusimos evaluar la efectividad de una protección física usando tubos de policloruro de vinilo (PVC) en la plantación de dos especies arbóreas nativas de las Sierras de Córdoba (Argentina) en un área con presencia de hormigas cortadoras. M\&M: Las especies de estudio fueron escallonia (Escallonia cordobensis) y maitén (Maytenus boaria). El área de plantación estuvo a $1840 \mathrm{~m} \mathrm{snm}$. Usamos 230 plantines por especie, 100 de ellos con y 130 sin protección de PVC. Fueron plantadas durante la estación húmeda en sitios con vegetación baja. Medimos semestralmente y por dos años la altura, la supervivencia y los daños por herbivoría.

Resultados: Para escallonia y maitén, respectivamente, la protección aumentó la supervivencia a los dos años en 24 y $26 \%$, aumentó la altura en 7,3 y $12,6 \mathrm{~cm}$, y redujo la herbivoría por hormigas cortadoras en 30 y $51 \%$ en comparación con los plantines sin protección.

Conclusiones: El efecto favorable de las protecciones en la supervivencia y crecimiento podría deberse conjuntamente a la disminución de la herbivoría por hormigas cortadoras y al resguardo contra las condiciones abióticas desfavorables. Recomendamos estudios de más años, y tendientes a entender los mecanismos que subyacen a la mejora en el establecimiento de los plantines.

\section{Palabras clave}

Acromyrmex lobicornis, Escallonia cordobensis, Maytenus boaria, reforestación, Sierras de Córdoba. 


\section{INTRODUCCIÓN}

Uno de los aspectos importantes para la restauración del bosque nativo es entender los efectos de la herbivoría sobre la regeneración de especies leñosas y cómo reducir los efectos negativos de la herbivoría en las especies de interés (Renison et al., 2015). La herbivoría por vertebrados puede tener efectos negativos en el establecimiento de las especies leñosas reduciendo su crecimiento y supervivencia en estadios tempranos de desarrollo a través del ramoneo y pisoteo (Howe, 1990; Torres et al., 2008, Renison et al., 2015). También la herbivoría por invertebrados puede afectar el establecimiento de renovales de especies leñosas ya que, por ejemplo, se ha registrado que pequeños daños foliares pueden causar efectos considerables en la producción fotosintética (Zangerl et al., 2002). A su vez, la existencia de selectividad en el consumo por parte de los herbívoros, tanto vertebrados como invertebrados, puede modificar la cobertura relativa de las especies en las comunidades vegetales (Hanley et al., 1995; Howe, 2008) o afectar el reclutamiento de plantas, determinando la presencia o ausencia de distintas especies en micro-hábitats específicos (Green et al., 1997; Rao et al., 2001).

Uno de los grupos de herbívoros invertebrados de mayor relevancia ecológica en América del Sur son las hormigas cortadoras ya que pueden consumir y movilizar una gran cantidad de biomasa vegetal, comparable a la cantidad consumida por grandes herbívoros (Hölldobler \& Wilson, 1990; Herrera \& Pellmyr, 2009; Montoya-Lerma et al., 2012). Estudios recientes en forestaciones de sauces (Salix spp.) y álamos (Populus spp.) mencionan daños por estas hormigas que alcanzan los $83 \mathrm{~kg} / \mathrm{ha}$ de material vegetal seco recolectado (Jiménez, 2019). Varios estudios realizados en bosques y sabanas tropicales y subtropicales han encontrado mayor densidad relativa de hormigueros en bosques degradados o bordes de bosque que en bosques conservados (Farji-Brener, 2001; Wirth et al., 2007; Siqueira et al., 2017). En estos sistemas considerablemente intervenidos por la acción humana, la herbivoría por hormigas podría modificar las trayectorias sucesionales que conducen al desarrollo de un bosque, sistema de referencia que usualmente se quiere alcanzar a través de las forestaciones con fines de restauración (Leal et al., 2014), en especial si se tiene en cuenta que las hormigas prefieren renovales arbóreos antes que las especies vegetales herbáceas (Herrera \& Pellmyr, 2009).

Estudios previos mencionan que la herbivoría por hormigas puede atenuarse plantando especies menos preferidas o que posean repelentes (Massad et al., 2011). Otros métodos propuestos incluyen: la exclusión física de las hormigas, el control mecánico de los hormigueros, el control biológico mediante patógenos o predadores y el uso de productos químicos tanto insecticidas como fungicidas (Montoya-Correa et al., 2007; Boulogne et al., 2012; Montoya-Lerma et al., 2012; Della Lucia et al., 2013). Sin embargo, a la hora de seleccionar un método para mitigar el efecto de la herbivoría por hormigas en las plantaciones, es necesario evaluar tanto las ventajas como las desventajas de cada método. Respecto a la utilización de plantas repelentes o más palatables que las de interés para restaurar, si se utiliza una especie exótica con capacidad de repelencia o palatabilidad (Delabie et al., 2003; Caffarini et al., 2008), se correría el riesgo de generar una invasión (Mack et al., 2000). Asimismo, no se ha encontrado información sobre características repelentes para hormigas cortadoras en especies vegetales nativas. En cuanto a la utilización de productos químicos, esto puede tener gran efectividad en el corto plazo, sin embargo, presenta desventajas por los daños ambientales que trae aparejados, como puede ser la mortalidad de especies que no son el objetivo de la aplicación (Della Lucia et al., 2013). Con respecto a la exclusión de las hormigas mediante algún tipo de barrera o protección física, tiene la ventaja de no introducir químicos dañinos en el ambiente al mismo tiempo que solo se necesitan conocimientos básicos del comportamiento de las hormigas, lo que puede hacer más sencilla su utilización (Jaacks, 2017). Varios métodos utilizados como barreras a las hormigas, como la colocación de grasa y sustancias pegajosas, tienen la desventaja que son de duración limitada y deben renovarse luego de lluvias o cuando se saturan (Montoya-Lerma et al., 2012). En un estudio 


\section{J. Arriaga et al. - Protección física y establecimiento de especies nativas}

previo en las Sierras de Córdoba se evaluó una técnica de protección física individual usando tubos de policloruro de vinilo (PVC) en plantaciones de Polylepis australis mostrando la potencialidad de ser efectiva para disuadir el ataque por hormigas cortadoras. Además, la técnica empleada tiene la ventaja de ser económica, durable, de bajo mantenimiento y ambientalmente segura (Jaacks, 2017).

Es de esperarse que técnicas como la protección física individual mediante tubos de PVC introduzca cambios micro-ambientales en el entorno del plantín. Algunos de estos cambios podrían ser, la menor incidencia del viento e insolación, la mayor retención de agua y temperatura alrededor del plantín. Se conoce que estas condiciones más favorables de micrositio pueden ser determinantes para el desempeño de los plantines (Schönbeck et al., 2015; Loranger et al., 2017; Sparacino et al., 2020). En sistemas en restauración similares a los del presente trabajo, aun sin presencia de hormigas cortadoras, se ha observado que las protecciones plásticas alrededor de plantines de Polylepis australis resultan beneficiosas para su establecimiento al protegerlos de los fuertes vientos presentes en la zona (Renison et al., 2002; Sparacino et al., 2020). En lo que respecta a las especies de interés para el presente trabajo, no se ha encontrado mayor información respecto a las condiciones óptimas de crecimiento para escallonia (Escallonia cordobensis), mientras que para maitén (Maytenus boaria) se menciona la posibilidad de que requiera protección por parte de la vegetación circundante para poder tolerar las condiciones abióticas desfavorables del área (Marro et al., 2017).

En este trabajo nos propusimos evaluar el efecto de una técnica de protección física (tubos de PVC) en el desempeño del establecimiento de plantines de maitén y escallonia plantados con fines de restauración en un sitio con presencia de hormigas cortadoras. Específicamente, comparamos la supervivencia, el crecimiento y el daño de la herbivoría por hormigas, sobre plantines con y sin protecciones físicas. Consideramos que la información autoecológica de las especies E. cordobensis y M. boaria es demasiado limitada como para aventurar sobre ellas una extrapolación de las conclusiones obtenidas en Polylepis australis (Jaacks, 2017). Además, no hemos encontrado estudios similares en ninguna otra especie. Debido a que las tres especies antes mencionadas (E. cordobensis, $M$. boaria y $P$. australis) son las más usadas en restauración de bosque en las Sierras Grandes de Córdoba, es de vital importancia optimizar sus técnicas de plantado y aumentar así la probabilidad de establecimiento.

\section{Materiales y Métodos}

\section{Área de estudio}

El estudio se realizó en las Sierras de Córdoba, en el centro de Argentina ( $31^{\circ} 34^{\prime} 0^{\prime}$ 'S; 6450'0' O; 500-2800 m). La temperatura media es de $11.2^{\circ} \mathrm{C}$ a $1800 \mathrm{msnm}$, existiendo temperaturas inferiores a $0^{\circ} \mathrm{C}$ durante todo el año (Marcora et al., 2008). La precipitación media anual fue de $900 \mathrm{~mm}$ en el período 19922010. La temporada seca y fría dura de mayo a septiembre y el $83 \%$ de la lluvia se concentra en los meses más cálidos entre octubre y abril (Colladon et al., 2010). Los suelos se derivan principalmente de la meteorización del sustrato de granito y depósitos eólicos de textura fina (Cabido et al., 1987).

La vegetación está constituida por un mosaico de pastizales, céspedes de pastoreo, bosques de Polylepis australis (donde se encuentran las especies leñosas de interés para el trabajo), afloramientos de granito y áreas erosionadas con superficies rocosas expuestas (Funes \& Cabido, 1995; Cingolani et al., 2003). La ganadería es la principal actividad económica de la zona, comenzó a principios del siglo XVII y reemplazó a los grandes herbívoros nativos a principios del siglo XX (Díaz et al., 1994).

Las plantaciones experimentales se realizaron en Los Gigantes, en un área de 19 ha (31 ${ }^{\circ} 23^{\prime} 12.79$ 'S; 644 4 '56.20"O) donde el ganado fue excluido en el año 2016. Esta área presenta una combinación de pastizales y afloramientos rocosos, en una altitud de 1840 msnm. Se estimó una densidad de hormigueros superior a 2 hormigueros/ha, densidad mayor a la estimada en el Parque Nacional Quebrada del Condorito de 0,66 hormigueros/ha (Renison, datos no publicados). La única especie de hormiga 
cortadora que detectamos en el área de estudio fue Acromyrmex lobicornis EMERY (1888) que tiene una distribución nativa Neotropical, incluyendo a Bolivia, Brasil, Paraguay, Uruguay y Argentina donde es la especie más abundante en zonas semiáridas (https://www.antwiki.org/ wiki/Acromyrmex_lobicornis, consulted April 2020).

\section{Especies seleccionadas}

La escallonia, Escallonia cordobensis (Kuntze) Hosseus (Escalloniaceae), es un arbusto o árbol pequeño, con múltiples fustes, de 1,5-3 $\mathrm{m}$ de altura, aunque puede alcanzar una altura de hasta $8 \mathrm{~m}$ (Renison observ. pers.). Esta especie es endémica de las Sierras Grandes, Sierras de los Comechingones y Sierras de San Luis, en las provincias de Córdoba y San Luis (Argentina). Crece en riberas de arroyos sobre suelos pedregosos, muchas veces asociados a los bosques de Polylepis. Se caracteriza por poseer tallos jóvenes densamente foliados, corteza gris claro a la base (Sede \& Denham, 2018).

El maitén, Maytenus boaria Molina (Celastraceae), es un árbol de alrededor de $8 \mathrm{~m}$ de altura, presente en los Andes de Argentina y Chile desde Tierra del Fuego/región Magallánica hasta la provincia de Mendoza/región de Atacama, en las sierras de San Luis y Córdoba; y en Brasil en las sierras de Río Grande Do Sul. Presenta reproducción por semillas, dispersadas por aves en los meses de verano (Demaio et al., 2015), además de reproducción vegetativa por raíz (Renison observ. pers.). Experimentos realizados en el centro de Argentina mostraron los mejores rendimientos de los plantines a campo entre los 1500 y $1800 \mathrm{msnm}$ y en sitios sin ganadería (Marcora et al., 2013).

\section{Diseño experimental}

Los plantines de maitén y escallonia fueron producidos en un vivero de la localidad de Cuesta Blanca (64 34' 46”O; $31^{\circ} 28^{\prime} 56^{\prime \prime}$ ) a partir de semillas recolectadas a $15 \mathrm{~km}$ o menos del área de plantación. Los plantines al momento del plantado tuvieron una edad aproximada de 18 meses y una altura promedio de $19 \mathrm{~cm}$ en maitén y de $34 \mathrm{~cm}$ en escallonia.

Se plantaron un total de 230 plantines de cada especie, en micrositios con vegetación baja (menor a $20 \mathrm{~cm}$ ) y con una separación entre plantines de al menos $3 \mathrm{~m}$. La plantación se hizo en enero de 2018, en hoyos de aproximadamente $35 \mathrm{~cm}$ de profundidad y $20 \mathrm{~cm}$ de diámetro cavados manualmente con pala, y los plantines se regaron por única vez en el momento de la plantación con 51 de agua. A un total de 100 plantines por especie seleccionados al azar, se les colocó una protección física y a 130 se los dejó sin protección. La razón para dejar un mayor número de plantines en el tratamiento control sin protección es que en experiencias previas este tratamiento tuvo una alta mortalidad (Jaacks, 2017) y es necesario tener un número inicial mayor para así poder tener suficientes plantines vivos para analizar el crecimiento.

La protección física consistió en un tubo de polivinilo de cloruro (PVC) colocado alrededor de cada plantín, como en Jaacks (2017). Las características de dichos tubos son: color blanco (impide el paso de la luz), $16 \mathrm{~cm}$ de diámetro y $30 \mathrm{~cm}$ de alto, siendo previsto que al momento de la plantación se los entierre en 5 o $7 \mathrm{~cm}$, quedando así una barrera física y visual de entre 20 a $25 \mathrm{~cm}$ de altura.

El monitoreo de los plantines se realizó cada 6 meses durante un periodo de 24 meses, teniéndose al final del experimento cuatro mediciones además de las condiciones iniciales. Para la elección de la cantidad y tiempo entre los monitoreos se tuvo en cuenta el trabajo previo de Jaacks (2017). Para evaluar el desempeño en el establecimiento, se registró la supervivencia y la altura de los plantines. Además, se registró la existencia de daño o corte en los plantines, diferenciando los daños ocasionados por hormigas de aquellos ocasionados por otros herbívoros mediante las características de los cortes que realizan los distintos herbívoros. Para el caso de las hormigas es común la defoliación total de los plantines dejando parte del tallo sin cortar, y cuando la defoliación no es total dejan cortes en forma de semicírculo (Bollazzi Sosa, 2014). A su vez, se tuvieron en cuenta las características de los cortes realizados por roedores y ungulados (descriptas en Schaller 2000) para distinguirlas de la realizadas por invertebrados. Se estimó el porcentaje del plantín que se encontraba defoliado. Como se observó que usualmente cuando las hormigas atacaban 


\section{J. Arriaga et al. - Protección física y establecimiento de especies nativas}

un plantín lo defoliaban en su totalidad (100\%), y con el objetivo de simplificar el análisis de los datos, se transformó la respuesta de esta variable a presencia/ausencia de herbivoría por hormigas.

\section{Análisis de datos}

Los datos se analizaron, por separado para cada especie, empleando Modelos Lineales Generalizados (MLG) usando el programa Infostat (Di Rienzo et al., 2017). Las variables de respuestas fueron la supervivencia y herbivoría por hormigas (presencia/ausencia) a los dos años de la plantación con asunción de distribución binomial y función de enlace logística, la altura final y el crecimiento total (altura final - altura inicial), con asunción de distribución normal y función de enlace identidad. Como variable predictora se incluyó el tratamiento de protección (con y sin protección). Además, para los plantines del tratamiento "sin protección", se analizó la supervivencia final a dos años del plantado (con asunción de distribución binomial) en función de la herbivoría por hormigas (con $\mathrm{y}$ sin signos de daño por hormigas durante el primer año). Se comprobaron los supuestos de normalidad y homocedasticidad para todos los análisis con variables de asunción de distribución normal.

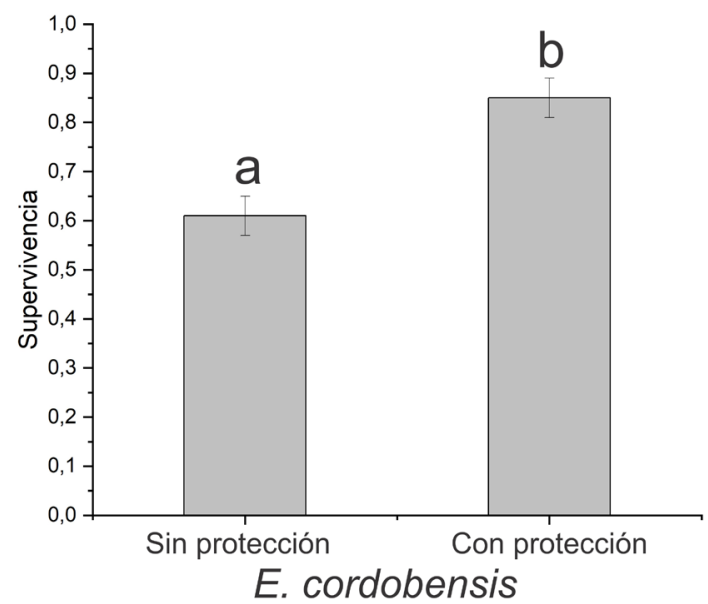

\section{Resultados}

A los dos años de plantados, la supervivencia de escallonia con y sin protección fue de $85 \%$ y $61 \%$, respectivamente, por lo que la protección aumentó la supervivencia en un 24\% (MLG: c2 = $15,2, \mathrm{GL}=1, \mathrm{P}<0,0001$; Fig. 1). Mientras que la supervivencia de maitén con y sin protección fue de $95 \%$ y $69 \%$, respectivamente, por lo que en este caso la protección aumentó la supervivencia en un 26\% (MLG: c2 $=18,5, \mathrm{GL}=1, \mathrm{P}<0,0001$; Fig. 1).

Para los plantines de escallonia, la altura promedio al final del experimento fue menor que al inicio del experimento. A los dos años de plantados, las escallonias con y sin protección, disminuyeron su altura $15 \mathrm{~cm}$ y 20 $\mathrm{cm}$, respectivamente $(\mathrm{MLG}: \mathrm{c} 2=5,2, \mathrm{GL}=$ $1, \mathrm{P}=0,022$; Fig. 2A), con una altura final de $19,8 \pm 1,5 \mathrm{~cm}(\mathrm{~N}=85)$ y $12,5 \pm 1,2 \mathrm{~cm}(\mathrm{~N}=$ 79), respectivamente (MLG: $\mathrm{c} 2=14,0, \mathrm{GL}=$ 1, P $<0,0001$; Fig. 2B). Por su parte, a los dos años de plantados, los maitenes con protección aumentaron en promedio $6 \mathrm{~cm}$ de altura, mientras que sin protección disminuyeron en promedio 8 cm (MLG: $\mathrm{c} 2=76,5, \mathrm{GL}=1, \mathrm{P}<0,0001$; Fig. $2 \mathrm{C})$, con una altura final de $24,0 \pm 1,4 \mathrm{~cm}(\mathrm{~N}=$ 95) y $11,4 \pm 0,9 \mathrm{~cm}(\mathrm{~N}=90)$, respectivamente (MLG: $\mathrm{c} 2=58,1, \mathrm{GL}=1, \mathrm{P}<0,0001$; Fig. 2D).

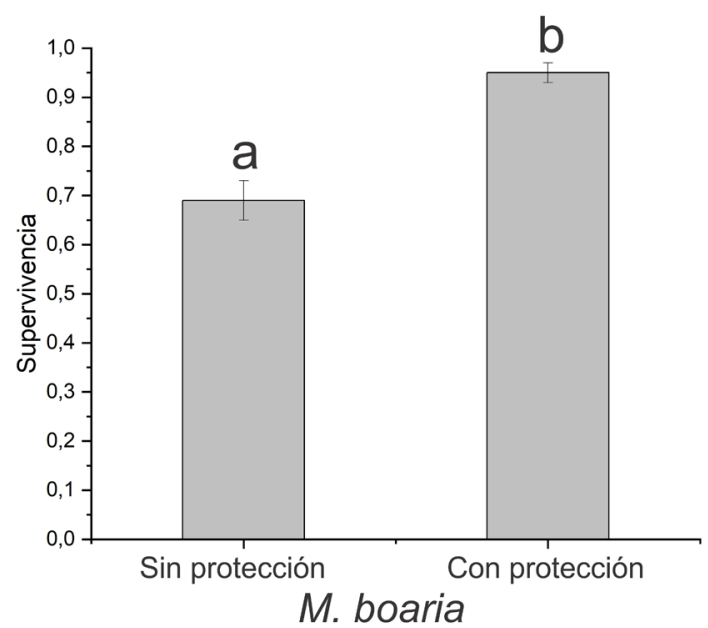

Fig. 1. Proporción de plantines vivos (supervivencia) en tratamientos con y sin protección para Escallonia cordobensis y Maytenus boaria, a los dos años de plantados. Las distintas letras indican diferencias significativas $(P<0,05)$. Las barras sobre las columnas indican error estándar. 

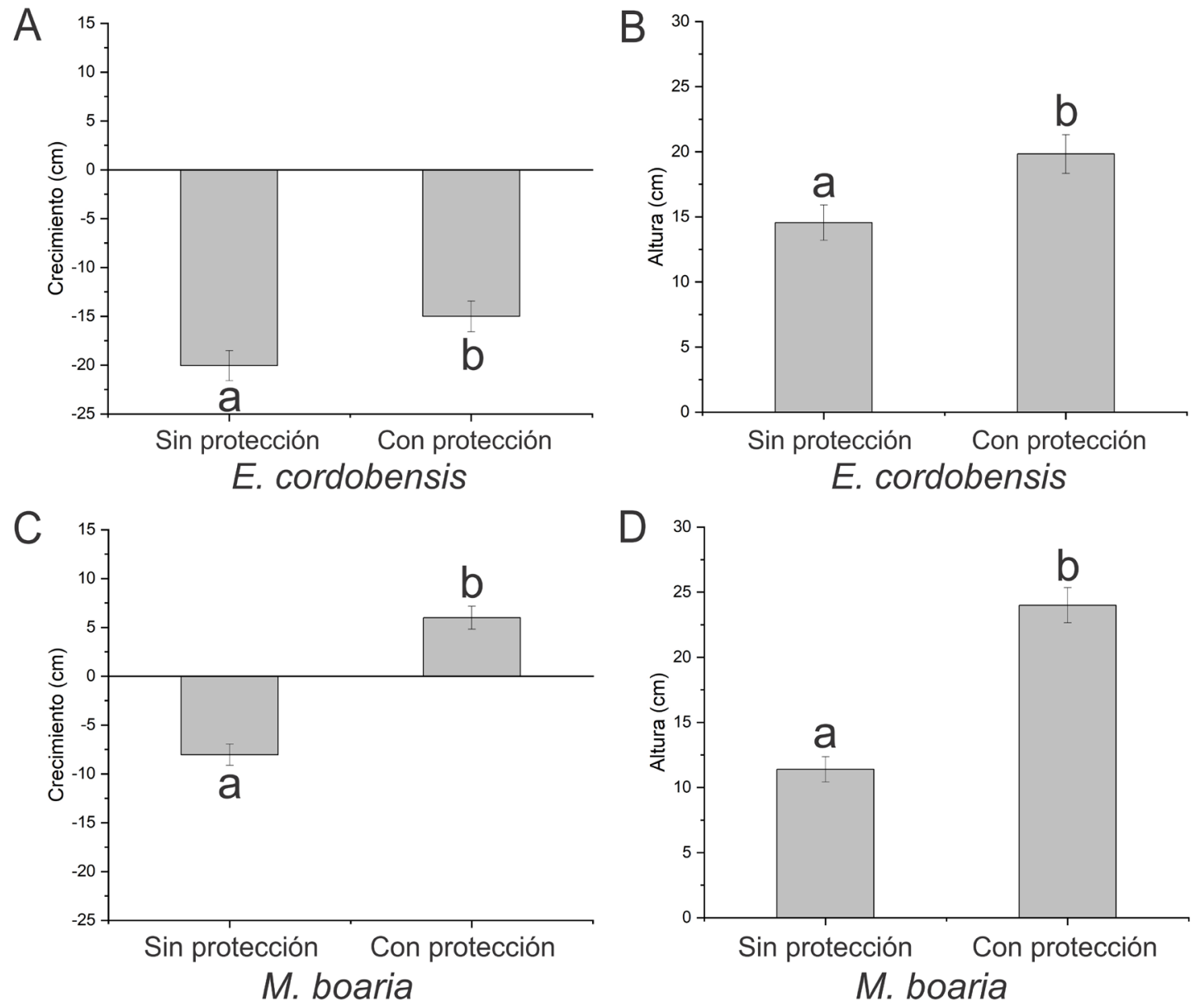

Fig. 2. Crecimiento $(\mathrm{cm})$ y altura media $(\mathrm{cm})$ en tratamientos con y sin protección para Escallonia cordobensis (A, B) y Maytenus boaria (C, D) a los dos años de plantados. Las distintas letras indican diferencias significativas $(P<0,05)$. Las barras sobre las columnas indican error estándar.

Al finalizar el experimento, el $42 \%$ del total de los plantines presentaron al menos una vez alguna clase de herbivoría, correspondiendo el $35 \%$ a herbivoría por hormigas cortadoras y el $7 \%$ restante a otros insectos o liebres. En las escallonias con y sin protección la proporción de plantines que sufrieron al menos un evento de herbivoría por hormigas fue de $21 \%$ y $51 \%$, respectivamente (MLG: $\mathrm{c} 2=25,7, \mathrm{GL}=1, \mathrm{P}<$ 0,0001 ; Fig. 3A), mientras que en los maitenes con y sin protección las cifras fueron de $4 \% \mathrm{y}$ $55 \%$, respectivamente (MLG: $\mathrm{c} 2=38,8, \mathrm{GL}$ $=1, \mathrm{P}<0,0001 ;$ Fig. 3B). La herbivoría por hormigas cortadoras varió a lo largo del tiempo, observándose mayor herbivoría en los registros de marzo y septiembre del primer año del experimento, siendo siempre mayor en los plantines sin protección (Fig. 3C).

En cuanto a la supervivencia de escallonia en función de la herbivoría por hormigas en plantines sin protección, la supervivencia a los dos años fue de $41 \%$ y $75 \%$ para los plantines con y sin signos de daño por hormigas durante el primer año, respectivamente. Así, la herbivoría por hormigas durante el primer año disminuyó la supervivencia a dos años en un 34\% (MLG: 

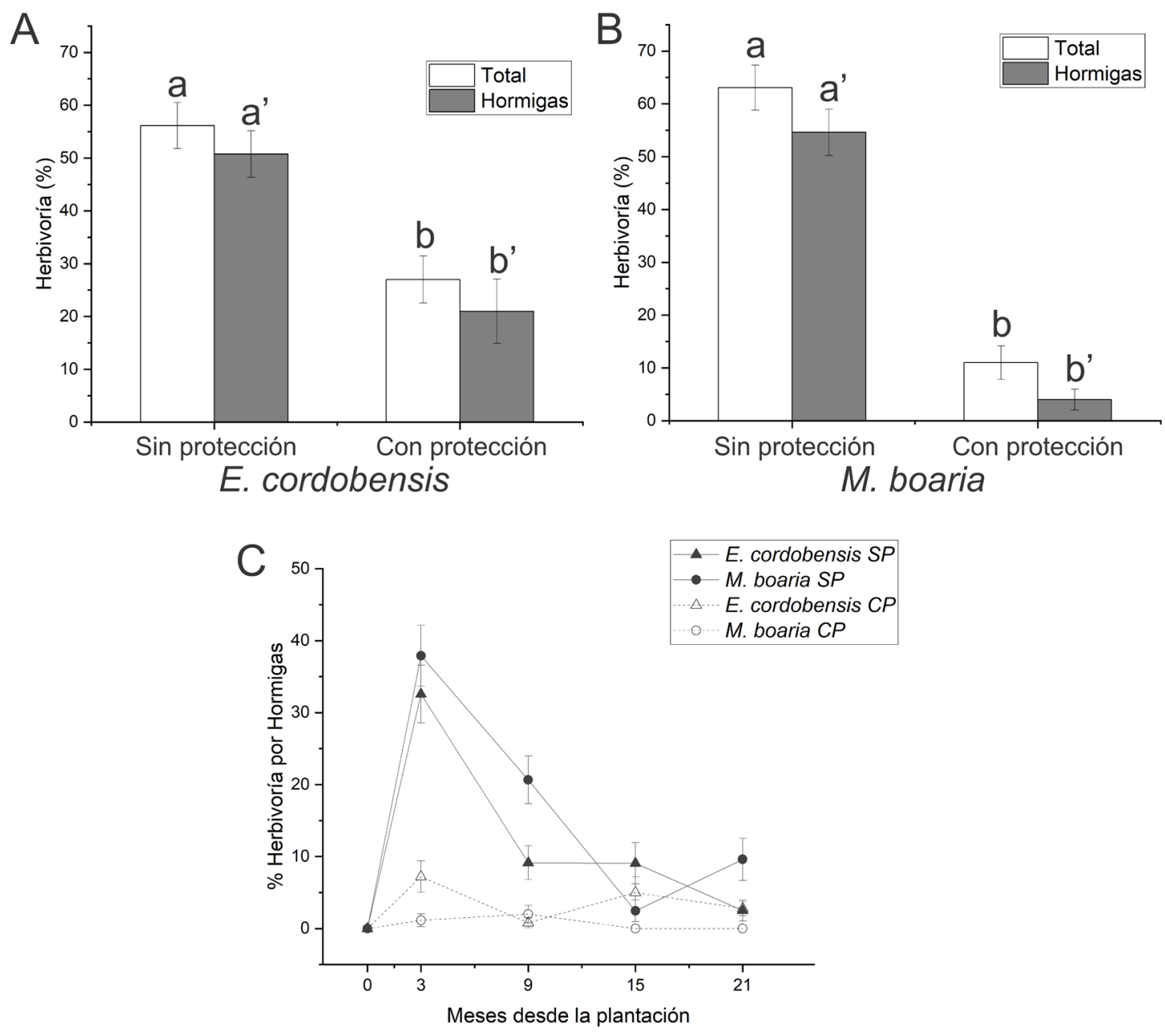

Fig. 3. Proporción de plantines en los que se registró herbivoría para A: escallonia (Escallonia cordobensis) y B: maitén (Maytenus boaria). Las columnas blancas representan la proporción de plantines que presentaron herbivoría de cualquier tipo (total) y las columnas grises representan la proporción de plantines que presentaron herbivoría por hormigas cortadoras. Las letras distintas indican diferencias significativas ( $P$ $<0,05)$ dentro del tipo de herbivoría considerados. Las barras sobre las columnas indican error estándar. C: Proporción de plantines que registraron herbivoría por hormigas cortadoras en función del tiempo después de la plantación. Líneas cortadas y símbolos vacíos representan los plantines con protección (CP). Líneas enteras y símbolos rellenos representan los plantines sin protección (SP). Símbolos triangulares representan los plantines de E. cordobensis. Símbolos circulares representan plantines de M. boaria. Las barras sobre los símbolos indican error estándar.

$\mathrm{c} 2=23,9, \mathrm{GL}=1, \mathrm{P}<0,0001$; Fig. 4). Para maitén, la supervivencia a los dos años fue de $51 \%$ y $87 \%$ en plantines con y sin signos de daño por hormigas durante el primer año, respectivamente. Así, la herbivoría por hormiga durante el primer año redujo la supervivencia a dos años en un 36\% (MLG: c2 $=35,5, \mathrm{GL}=1$, $\mathrm{P}<0,0001$; Fig. 4). No se realizó este mismo análisis para los plantines con protección debido a que en estos plantines se registró muy poca herbivoría por hormigas durante el primer año $(8$ plantines de escallonia y 4 de maitén). 

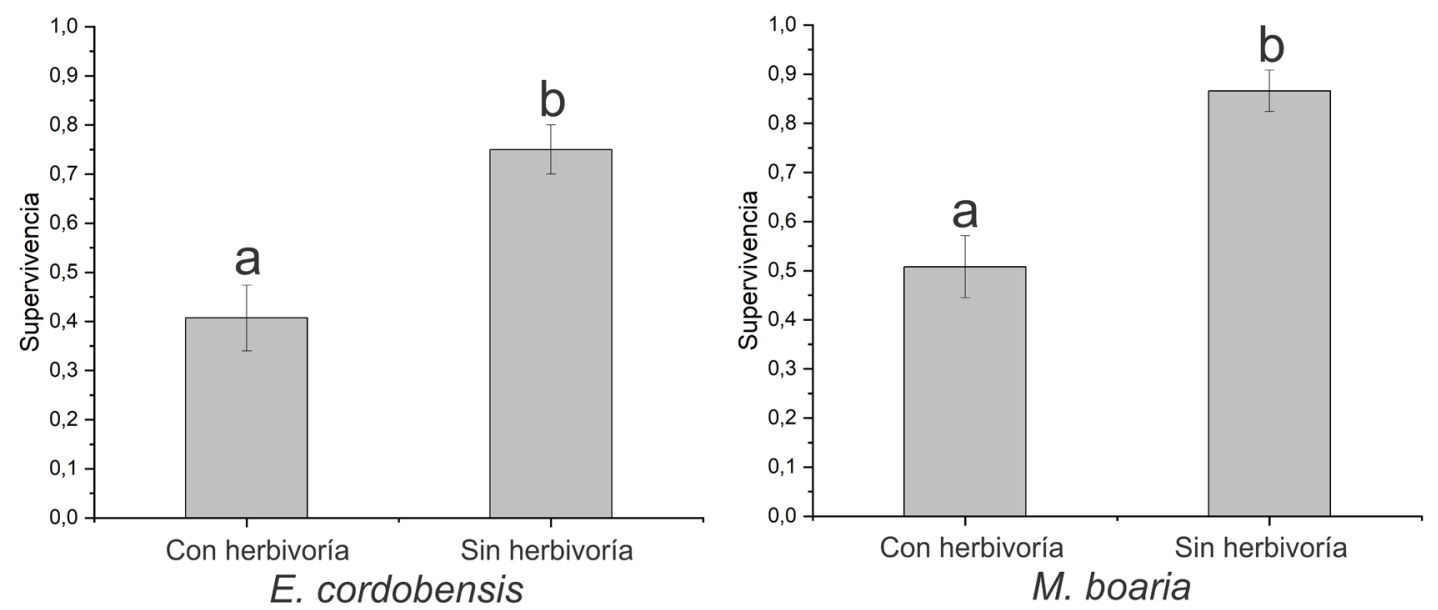

Fig. 4. Proporción de plantines vivos (supervivencia) en condiciones con o sin presencia de herbivoría por hormigas durante el primer año desde la plantación, para Escallonia cordobensis y Maytenus boaria, a los dos años de plantados. Las distintas letras indican diferencias significativas $(P<0,05)$. Las barras sobre las columnas indican error estándar.

\section{Discusión y ConClusiones}

Nuestros resultados muestran una mayor supervivencia y crecimiento para los plantines con protección de escallonia y maitén (E. cordobensis y $M$. boaria), en concordancia con el trabajo previo con la especie Polylepis australis (Jaacks, 2017). El menor daño por hormigas registrado parece explicar en gran parte la mejora en el desempeño de los plantines con protección, aunque otros factores, como las modificaciones micro-ambientales que posiblemente introducen las protecciones, también podrían influir en este resultado.

Según estudios en medianos y grandes herbívoros, los sistemas de montaña pueden ser altamente susceptibles al efecto que genera la herbivoría sobre la estructura de la vegetación (Hofgaard et al., 2010; Speed et al., 2011). En particular en las Sierras de Córdoba se reconoce un fuerte efecto de simplificación de los bosques de $P$. australis por parte de la herbivoría por ganado doméstico (Cingolani et al., 2003, 2008; Renison et al., 2011, 2015), pero poco se sabe del efecto de la herbivoría por invertebrados, y en particular por hormigas, en estos sistemas. Aún se necesitan más estudios para entender en profundidad los mecanismos que subyacen el efecto positivo de las protecciones físicas en el desempeño de los plantines. Sin embargo, los resultados aquí obtenidos muestran que, al menos en algunos sitios degradados, la herbivoría por hormigas puede condicionar el éxito de las plantaciones y del establecimiento natural de leñosas, como sucede con el ganado doméstico.

El efecto de la herbivoría por hormigas en áreas en proceso de restauración ha sido observado en varias regiones del neotrópico, ya que por ejemplo, las hormigas suelen predar o remover las semillas introducidas o bien atacar las hojas nuevas de plantas jóvenes (Majer et al., 2007). Diversos estudios ilustran los severos daños que las hormigas cortadoras generan tanto en plantaciones forestales con fines productivos (Pérez et al., 2011; Nickele et al., 2012; Zanetti et al., 2014), como también en reforestaciones con plantas nativas (Garen et al., 2009; Perin \& Guimarães, 2012). Estos daños se concentran en los primeros años de regeneración del bosque (Vasconcelos \& Cherrett, 1997). A su vez, existe una preferencia de las hormigas cortadoras por bosques degradados o bordes de bosques (Farji-Brener, 2001; Wirth et al., 2007; Meyer, 2008; Barrera et al., 2015; Siqueira et al., 2017; Amaya-Hernández et al., 2019), condición que caracteriza las etapas iniciales de los sitios de restauración de las Sierras de Córdoba.

En concordancia con nuestros resultados, experiencias previas de exclusión de hormigas 


\section{J. Arriaga et al. - Protección física y establecimiento de especies nativas}

cortadoras a través de una barrera física, han mostrado un aumento en la supervivencia y una disminución de la herbivoría por hormigas en los plantines protegidos (Vasconcelos \& Cherrett, 1997; Pérez et al., 2011; Jaacks, 2017). Los antecedentes mencionados y los resultados del presente trabajo refuerzan la idea de que, al menos parcialmente, la mayor supervivencia presentada por los plantines con protección se debe a una menor frecuencia de herbivoría por hormigas cortadoras. Considerando la limitada información disponible sobre la autoecología de las especies estudiadas, escallonia y maitén, el presente trabajo aportó evidencia sobre la susceptibilidad que presentan ambas especies a la herbivoría por hormigas.

La diferencia de crecimiento entre los plantines con y sin protección puede también verse explicada por el resguardo contra condiciones climáticas extremas (frío y viento) que generaron las protecciones. En otras especies las heladas causan la muerte de la parte alta de los plantines y generan una disminución en la altura promedio (Torres \& Renison, 2015; Verzino et al., 2004; Körner \& Paulsen, 2004; Marcora et al., 2013). Apoyando una hipótesis de disminución de altura por heladas en el presente estudio, se observó en las mediciones posteriores al invierno que algunos plantines se encontraban secos en la porción de la planta que quedaba por fuera de la protección y que por dentro de las mismas presentaban sus hojas en buen estado de salud (observ. pers.). También se ha observado en experimentos con P. australis, que las protecciones plásticas aumentaron el crecimiento de los plantines, aunque no la supervivencia, aparentemente por generar una protección contra el viento (Renison et al., 2002; Sparacino et al., 2020). No contar con valores de alguna de las variables ambientales dentro y fuera de las protecciones fue una limitación del presente trabajo. Lo cual impidió profundizar en los mecanismos mediante los cuales las protecciones estarían aumentando el desempeño en el establecimiento.

Es notable el mayor crecimiento del maitén ( $M$. boaria) plantado con protección, en comparación con los plantados sin protección (Fig. 2C) y en comparación con el crecimiento reportado en los estudios previos hechos en la zona (Marcora et al., 2013; Marro et al., 2017). Esta diferencia en el crecimiento parece deberse al resguardo generado por las protecciones contra las condiciones ambientales desfavorables que prevalecen en la zona. Experiencias previas con maitén registran crecimiento recién a partir del quinto año desde la plantación, hipotetizándose que transcurrido ese tiempo la vegetación circundante podría estar facilitando el crecimiento por un efecto nodriza (Marro et al., 2017). Esta explicación hipotética también se apoya en el hecho de que en el centro de Argentina el maitén a menudo sólo se encuentra en bosques maduros (Renison et al., 2011). En el presente trabajo, el crecimiento positivo del maitén en los primeros dos años desde la plantación parece asociarse a que las protecciones generan un resguardo contra condiciones abióticas hostiles, similar al que posibilita la vegetación circundante, en plazos temporales más amplios o en bosques conservados.

Costos y beneficios del uso de protecciones y sus implicancias para la reforestación

Las protecciones físicas aumentaron el desempeño en el establecimiento de los plantines de escallonia y maitén. Sin embargo, la utilización de las protecciones aumentó el costo de plantación de USD \$ 0,8 (Renison, comunicación personal) a USD $\$ 3,0$ por plantín, sin considerar la planificación que debe realizarse a medio o largo plazo respecto de los residuos que generan las protecciones cuando ya no son utilizables. Ponderando la altura de los plantines, su supervivencia y el costo como en Jaacks (2017) se obtiene que la eficiencia de plantación luego de dos años fue un $46 \%$ mejor sin protección. Si bien la diferencia monetaria no parece justificar la colocación de protecciones y las consecuentes mejoras en el desempeño de los plantines, antes de descartar la técnica completamente deberían evaluarse posibles mejoras. Por ejemplo, la reutilización de las protecciones en futuras plantaciones podría disminuir costos, además de considerarse que su rédito podría ser mayor a más años y que en sitios degradados a ciertas altitudes estas protecciones podrían ser la única forma de garantizar el éxito de la reforestación. En lo que respecta particularmente al daño por hormigas cortadoras, podría evaluarse la utilización simultánea de otras técnicas de bajo costo, como la plantación adjunta de plantas repelentes o más palatables también nativas de la zona, o la destrucción mecánica de los hormigueros con el fin de reducir la herbivoría. 


\section{Contribución DE LOS AUTORES}

JA y DR concibieron la idea. JA y DR concretaron el experimento a campo. JA realizó los monitoreos. JA, DR y RT analizaron los datos. JA elaboró el manuscrito. Todos los autores discutieron los resultados e hicieron comentarios sobre el manuscrito.

\section{Agradecimientos}

A Ronaldo H. Renison por ayudar tanto en la producción de los arbolitos. Deutsche Forschungsgemeinschaft Grant (DFG-Alemania) y Consejo Nacional de Investigaciones Científicas y Técnicas (CONICET-Argentina) proveyeron los fondos. La Familia Ruiz Moreno nos permitió realizar este estudio en su campo y nos brindó alojamiento. Julio Domínguez facilitó los contactos, construyó los alambrados y ayudó en las plantaciones. Numerosos voluntarios ayudaron durante las plantaciones. Dos revisores anónimos y el editor a cargo mejoraron sustancialmente la redacción del manuscrito.

\section{Biblografía}

AMAYA-HERNÁNDEZ, L. M., Y. A. MERAVELASCO, M. C. GALLEGO-ROPERO, J. MONTOYA-LERMA \& I. ARMBRECHT. 2019. Herbivoría de Atta cephalotes (Hymenoptera: Formicidae) en parches de bosque seco tropical del sur-occidente colombiano. Bol. Cient. Mus. Hist. Nat. U. de Caldas, 23: 171-189.

http://dx.doi.org/10.17151/bccm.2019.23.2.9

BARRERA, C. A., L. M. BUFFA \& G. VALLADARES. 2015. Do leaf-cutting ants benefit from forest fragmentation? Insights from community and species-specific responses in a fragmented dry forest. Insect Conserv. Divers. 8: 456-463. https://doi.org/10.1111/icad.12125

BOLLAZZI SOSA, M. 2014. Hormigas cortadoras de hojas Acromyrmex spp. Reconocimiento a campo de plagas y enfermedades forestales. INIA, Instituto Nacional de Investigación Agropecuaria, Uruguay. Cartilla $\mathrm{n}^{\circ} 36$.

BOULOGNE, I., P. PETIT, H. OZIER-LAFONTAINE, L. DESFONTAINES \& G. LORANGERMERCIRIS. 2012. Insecticidal and antifungal chemicals produced by plants: a review. Environ. Chem. Lett. 10: 325-347.

https://doi.org/10.1007/s10311-012-0359-1

CABIDO, M., R. BREIMER \& G. VEGA. 1987. Plant communities and associated soil types in a high plateau of the Córdoba mountains, central Argentina. Mt. Res. Dev. 7: 25-42. https://doi.org/10.2307/3673322

CAFFARINI, P., P. CARRIZO, A. PELICANO, P. ROGGERO, \& J. PACHECO. 2008. Efectos de extractos acetónicos y acuosos de Ricinus communis (ricino), Melia azedarach (paraíso) y Trichillia glauca (trichillia), sobre la hormiga negra común (Acromyrmex lundi). Idesia (Arica) 26: 59-64. http://dx.doi.org/10.4067/S0718-34292008000100008

CINGOLANI, A. M., M. R. CABIDO, D. RENISON \& V. SOLÍS NEFFA. 2003. Combined effects of environment and grazing on vegetation structure in Argentine granite grasslands. J. Veg. Sci. 14: $223-$ 232.

https://doi.org/10.1111/j.1654-1103.2003.tb02147.x

CINGOLANI, A. M., D. RENISON, P. A. TECCO, D. E. GURVICH \& M. CABIDO. 2008. Predicting cover types in a mountain range with long evolutionary grazing history: a GIS approach. J. Biogeogr. 35: 538-551.

https://doi.org/10.1111/j.1365-2699.2007.01807.x

COLLADON, L., G. FELICI \& I. PAZOS. 2010. Anuario pluviométrico 205/ 06 2009/10 de la Cuenca del Río San Antonio. [online]. Disponible en: https://www. argentina.gob.ar/ina [Acceso: 20 Marzo 2021].

DELABIE, J. H. C., M. OSPINA \& G. ZABALA. 2003. Relaciones entre hormigas y plantas: una introducción. En: FERNANDEZF. (ed.) Introducción a las Hormigas de la Región Neotropical, pp 167-180. Instituto de investigación de recursos biológicos Alexander von Humboldt, Bogotá.

DELLA LUCIA, T. M., L. C. GANDRA \& R. N. GUEDES. 2013. Managing leaf-cutting ants: peculiarities, trends and challenges. Pest Manag. Sci. 70: 14-23. https://doi.org/10.1002/ps.3660

DEMAIO, P. H., M. MEDINA y U. KARLIN. 2015. Árboles Nativos De Argentina. Tomo 1: Centro y Cuyo. Ecoval Editorial, Córdoba.

DÍAZ, S., A. ACOSTA \& M. CABIDO. 1994. Community structure in montane grasslands of central Argentina in relation to land use. Appl. Veg. Sci. 5: 483-488. https://doi.org/10.2307/3235974

DI RIENZO, J. A., MACCHIAVELLI, R. \& CASANOVES, F. 2017. Modelos lineales 


\section{J. Arriaga et al. - Protección física y establecimiento de especies nativas}

generalizados mixtos aplicaciones en InfoStat. Grupo Infostat, Córdoba, Argentina [online]. Disponible en: https://www.researchgate.net/profile/Fernando Casanoves/publication [Acceso: 7 Febrero 2021].

FARJI-BRENER, A. G. 2001. Why are leaf-cutting ants more common in early secondary forests than in old-growth tropical forests? An evaluation of the palatable forage hypothesis. Oikos 92: 169-177. https://doi.org/10.1034/j.1600-0706.2001.920120.x

FUNES, G. \& M. CABIDO. 1995. Variabilidad local y regional de la vegetación rupícola de las Sierras Grandes de Córdoba, Argentina. Kurtziana 24: 173-173.

GAREN, E. J., K. SALTONSTALL, J. L. SLUSSER, S. MATHIAS, M. S. ASHTON \& J. S. HALL. 2009. An evaluation of farmers' experiences planting native trees in rural Panama: implications for reforestation with native species in agricultural landscapes. Agrofor. Syst. 76: 219-236. https://doi.org/10.1007/s10457-009-9203-4

GREEN, P. T., D. J. O'DOWD \& P. S. LAKE. 1997. Control of seedling recruitment by land crabs in rain forest on a remote oceanic island. Ecology 78: 2474-2486. https://doi.org/10.1890/00129658(1997)078[2474:COSRBL]2.0.CO;2

HANLEY, M. E., M. FENNER \& P. J. EDWARDS. 1995. An experimental field study of the effects of mollusc grazing on seedling recruitment and survival in grassland. J. Ecol. 83: 621-627.

https://doi.org/10.2307/2261630

HERRERA, C. M. \& O. PELLMYR. (Eds.). 2009. Plant animal interactions: an evolutionary approach. John Wiley \& Sons.

HOFGAARD, A., J. O. LØKKEN, L. DALEN \& H. HYTTEBORN. 2010. Comparing warming and grazing effects on birch growth in an alpine environment-a 10-year experiment. Plant Ecol. Divers. 3: 19-27. https://doi.org/10.1080/17550871003717016

HÖLLDOBLER, B. \& E. O. WILSON. 1990. The ants. Harvard University Press.

HOWE, H. F. 1990. Survival and growth of juvenile Virola surinamensis in Panama: effects of herbivory and canopy closure. J. Trop. Ecol, $6: 259-280$. https://www.jstor.org/stable/2559826

HOWE, H. F. 2008. Reversal of fortune: plant suppression and recovery after vole herbivory. Oecologia 157 : 279-286.

https://doi.org/10.1007/s00442-008-1069-z

JAACKS, G. 2017. Evaluación de una técnica contra hormigas para el proyecto de reforestación en el
Parque Nacional Quebrada del Condorito. Tesis de maestría. Universidad Nacional de Córdoba, Argentina.

JIMÉNEZ, N. L. 2019. Patrones de herbivoría y coocurrencia de hormigas cortadoras de hojas en forestaciones y áreas naturales del Bajo Delta del Río Paraná, Argentina. Tesis de doctorado. Universidad de Buenos Aires, Argentina.

KÖRNER, C. \& J. PAULSEN. 2004. A world-wide study of high altitude treeline temperatures. J. Biogeogr. 31: 713-732. https://doi.org/10.1111/j.1365-2699.2003.01043.x

LEAL, I. R., R. WIRTH \& M. TABARELLI. 2014. The multiple impacts of leaf-cutting ants and their novel ecological role in human-modified neotropical forests. Biotropica 46: 516-528.

https://doi.org/10.1111/btp.12126

LORANGER, H., G. ZOTZ \& M. Y. BADER. 2017. Competitor or facilitator? The ambiguous role of alpine grassland for the early establishment of tree seedlings at treeline. Oikos 126: 1625-1636.

https://doi.org/10.1111/oik.04377

MACK, R. N., D. SIMBERLOFF, W. MARK LONSDALE, H. EVANS, M. CLOUT, \& F. A. BAZZAZ. 2000. Biotic invasions: causes, epidemiology, global consequences, and control. Ecol. Appl. 10: 689-710. https://doi.org/10.1890/10510761(2000)010[0689:BICEGC]2.0.CO;2

MAJER, J. D., K. E. BRENNAN \& M. L. MOIR. 2007. Invertebrates and the restoration of a forest ecosystem: 30 years of research following bauxite mining in Western Australia. Restor. Ecol. 15: S104-S115. https://doi.org/10.1111/j.1526-100X.2007.00298.x

MARCORA, P., I. HENSEN, D. RENISON, P. SELTMANN \& K. WESCHE. 2008. The performance of Polylepis australis trees along their entire altitudinal range: implications of climate change for their conservation. Divers. Distrib. 14: 630-636.

https://doi.org/10.1111/j.1472-4642.2007.00455.x

MARCORA, P. I., D. RENISON, A. I. PAÍS-BOSCH, M. R. CABIDO \& P. A. TECCO. 2013. The effect of altitude and grazing on seedling establishment of woody species in central Argentina. Forest Ecol. Manag. 291: 300-307.

https://doi.org/10.1016/j.foreco.2012.11.030

MARRO, N., F. SOTERAS, N. COFRÉ, I. IBARRA, R. TORRES, A. G. BECERRA \& D. RENISON. 2017. Neotropical tree production: insights into 
germination, growth and outplanting for Maytenus boaria. Cerne 23 : 377-385.

https://doi.org/10.1590/01047760201723032344

MASSAD, T. J., J. Q. CHAMBERS, S. G. ROLIM, R. M. JESUS, \& L. A. DYER. 2011. Restoration of pasture to forest in Brazil's Mata Atlântica: The roles of herbivory, seedling defenses, and plot design in reforestation. Restor. Ecol. 19: 257-267. https://doi.org/10.1111/j.1526-100X.2010.00683.x

MEYER, S. T. 2008. Ecosystem engineering in fragmented forests: edge-mediated hyperabundance of leaf-cutting ants and resulting impacts on forest structure, microclimate and regeneration. Doktorarbeit. Universität Kaiserslautern.

MONTOYA-CORREA, M., J. MONTOYA-LERMA, I. ARMBRECHT \& M. C. GALLEGO-ROPERO. 2007. ¿Cómo responde la hormiga cortadora de hojas Atta cephalotes (Hymenoptera: Myrmicinae) a la remoción mecánica de sus nidos? Bol. Mus. Ent. Univ. Valle 8: 1-8.

MONTOYA-LERMA, J., C. GIRALDO-ECHEVERRI, I. ARMBRECHT, A. FARJI-BRENER \& Z. CALLE. 2012. Leaf-cutting ants revisited: towards rational management and control. Int. J. Pest Manag. 58: 225247. https://doi.org/10.1080/09670874.2012.663946

NICKELE, M. A., W. REIS FILHO, E. B. D. OLIVEIRA, E. T. IEDE, N. CALDATO \& P. STRAPASSON. 2012. Leaf-cutting ant attack in initial pine plantations and growth of defoliated plants. Pesqui. Agropecu. Bras. 47: 892-899.

https://doi.org/10.1590/S0100-204X2012000700003

PÉREZ, S. P., J. C. CORLEY \& A. G. FARJI-BRENER. 2011. Potential impact of the leaf-cutting ant Acromyrmex lobicornis on conifer plantations in northern Patagonia, Argentina. Agric. Forest Entomol. 13: 191-196. https://doi.org/10.1111/j.1461-9563.2010.00515.x

PERIN, M. A. A. \& J. F. GUIMARÃES. 2012. Efeitos dos ninhos de Atta laevigata (Fr. Smith, 1858) (Hymenoptera: Formicidae) sobre a vegetação do cerrado. Rev. Arvore 36: 463-470. http://dx.doi.org/10.1590/S0100-67622012000300008

RAO, M., J. TERBORGH \& P. NUÑEZ. 2001. Increased herbivory in forest isolates: implications for plant community structure and composition. Conserv. Biol. 15: 624-633. https://doi.org/10.1046/j.1523-1739.2001.015003624.x

RENISON, D., A. M. CINGOLANI \& D. SCHINNER. 2002. Optimizing restoration of Polylepis australis woodlands: when, where and how to transplant seedlings to the mountains. Ecotropica 8: 219-224.

RENISON, D., I. HENSEN \& R. SUAREZ. 2011. Landscape structural complexity of high-mountain Polylepis australis forests: a new aspect of restoration goals. Restor. Ecol., 19, 390-398. https://doi.org/10.1111/j.1526-100X.2009.00555.x

RENISON, D., M. P. CHARTIER, M. MENGHI, P. I. MARCORA, R. C. TORRES, M. GIORGIS \& A. M. CINGOLANI. 2015. Spatial variation in tree demography associated to domestic herbivores and topography: Insights from a seeding and planting experiment. Forest Ecol. Manag. 335: 139-146. https://doi.org/10.1016/j.foreco.2014.09.036

SCHALLER, M. J. 2000. Evaluation of wildlife damage to forests in Germany. Human Conflicts with Wildlife: Economic Considerations 14: 123-126.

SCHÖNBECK, L., M. LOHBECK, F. BONGERS, M. M. RAMOS \& F. Sterck. 2015. How do light and water acquisition strategies affect species selection during secondary succession in moist tropical forests?. Forests 6: 2047-2065.

https://doi.org/10.3390/f6062047

SEDE, S. M. \& S. S. DENHAM. 2018. Taxonomic Revision of Escallonia (Escalloniaceae) in Argentina. Syst. Bot. 43: 364-396.

https://doi.org/10.1600/036364418X696932

SIQUEIRA, F. F., J. D. RIBEIRO-NETO, M. TABARELLI, A. N. ANDERSEN, R. WIRTH \& I. R. LEAL. 2017. Leaf-cutting ant populations profit from human disturbances in tropical dry forest in Brazil. J. Trop. Ecol. 33: 337-344. https://doi.org/10.1017/S0266467417000311

SPARACINO, J., D. RENISON, A. M. DEVEGILI \& R. SUAREZ. 2020. Wind protection rather than soil water availability contributes to the restriction of high-mountain forest to ravines. New For. 51: 101117. https://doi.org/10.1007/s11056-019-09722-z

SPEED, J. D., G. AUSTRHEIM, A. J. HESTER \& A. MYSTERUD. 2011. Growth limitation of mountain birch caused by sheep browsing at the altitudinal treeline. Forest Ecol. Manag., 261, 1344-1352. https://doi.org/10.1016/j.foreco.2011.01.017

TORRES, R. C., RENISON, D., HENSEN, I., SUAREZ, R., ENRICO, L. 2008. Polylepis australis' regeneration niche in relation to seed dispersal, site characteristics and livestock density. Forest Ecol. Manag. 254: 255-260.

TORRES, R. C. \& D. RENISON. 2015. Effects of vegetation and herbivores on regeneration of two 


\section{J. Arriaga et al. - Protección física y establecimiento de especies nativas}

tree species in a seasonally dry forest. J. Arid Environ. 121: 59-66.

https://doi.org/10.1016/j.jaridenv.2015.05.002

VASCONCELOS, H. L. \& J. M. CHERRETT. 1997.

Leaf-cutting ants and early forest regeneration in central Amazonia: effects of herbivory on tree seedling establishment. J. Trop. Ecol. 13: 357-370. https://www.jstor.org/stable/2560289

VERZINO, G., J. JOSEAU, M. DÍAZ \& M. DORADO. 2004. Comportamiento inicial de especies nativas del Chaco Occidental en plantaciones en zonas de pastizales de altura de las Sierras de Córdoba, Argentina. Bosque (Valdivia) 25: 53-67. http://dx.doi.org/10.4067/S0717-92002004000100005

WIRTH, R., S. T. MEYER, W. R. ALMEIDA, M. V. ARAÚJO JR, V. S. BARBOS, \& I. R. LEAL. 2007.
Increasing densities of leaf-cutting ants (Atta spp.) with proximity to the edge in a Brazilian Atlantic forest. J. Trop. Ecol. 23: 501-505. https://www.jstor. org/stable/4499125

ZANETTI, R., J. C. ZANUNCIO, J. C. SANTOS, W. L. P. DA SILVA, G. T. RIBEIRO \& P. G. LEMES. 2014. An overview of integrated management of leaf-cutting ants (Hymenoptera: Formicidae) in Brazilian forest plantations. Forests 5: 439-454. https://doi.org/10.3390/f5030439

ZANGERL, A. R., J. G. HAMILTON, T. J. MILLER, A. R. CROFTS, K. OXBOROUGH, M. R. BERENBAUM \& E. H. DE LUCIA. 2002. Impact of folivory on photosynthesis is greater than the sum of its holes. Proc. Natl. Acad. Sci. 99: 1088-1091. https://doi.org/10.1073/pnas.022647099 
